\title{
Atypical bullous pyoderma gangrenosum associated with myeloid malignancies
}

\author{
C R M HAY,* A G MESSENGER, $\dagger$ D W K COTTON, $\ddagger$ S S BLEEHEN, $\dagger$ \\ D A WINFIELD*
}

From the University Departments of ${ }^{*}$ Haematology, $\ddagger$ Pathology, and $†$ Dermatology, Royal Hallamshire

Hospital, Sheffield

SUMMARY Atypical bullous pyoderma gangrenosum was diagnosed during the course of a myeloid malignancy in three patients. One patient had chronic myeloid leukaemia, one acute myeloid leukaemia, and the third, refractory anaemia with excess of blasts. This atypical form of pyoderma gangrenosum has been specifically associated with myeloid malignancies. The atypical appearance of the skin lesions and the clinical context in which they arose caused the true diagnosis to be delayed in all cases. Treatment with steroids was associated with rapid healing of the skin lesion. The histopathological changes in the skin biopsy specimens from these cases were non-specific, and although the histology was considered to be atypical of pyoderma gangrenosum in one case, the unusual features could be attributed to the patient's neutropenia. (Skin biopsy was performed to exclude other specific pathology).

Atypical bullous pyoderma gangrenosum is an uncommon association of the myeloid malignancies. It may remain unrecognised and should be considered more often.

Pyoderma gangrenosum is a clinical diagnosis largely based on the appearance of the typical lesion. This has a raised plaque with progressive central necrosis and a bluish-red necrotising border. A rapid response to steroids lends further support to the diagnosis and is thought to be an essential diagnostic criterion by some authors. ${ }^{1}$ Although a skin biopsy specimen is usually obtained, it does not provide independent confirmation of the diagnosis. The histopathological features are non-specific and variable. Some features remain in dispute ${ }^{2-4}$; the presence of vasculitis, in particular, is thought by some authors to exclude pyoderma gangrenosum. ${ }^{5}$

Fifty per cent of cases are associated with bowel disease ${ }^{4}$ and when seen in this clinical context, the diagnosis is easy. Diagnostic difficulty may arise in the absence of systemic disease or with less well known associations such as myeloid malignancies. Pyoderma gangrenosum has been observed in patients with myeloproliferative disorders, ${ }^{45}$ myelodysplasia, ${ }^{16}$ and acute myeloid leukaemia. ${ }^{47}$ Though it has been associated with blast crisis and poor prognosis, it may arise at any stage of the dis-

Accepted for publication 6 October 1986 ease, even as the presenting complaint. ${ }^{7}$ As skin lesions in myeloid malignancies are usually caused by haemorrhage, infection, or leukaemia cutis the true diagnosis of pyoderma gangrenosum may be delayed or missed. Additional problems may arise because the atypical bullous variant of pyoderma gangrenosum associated with myeloid malignancies ${ }^{148}$ is often unfamiliar and may not be recognised.

We describe three cases presenting over 12 months to draw attention to the association between pyoderma gangrenosum and the myeloid malignancies, and also to illustrate the characteristic clinical features and natural history. The value of skin biopsy in this situation and the range of histopathological changes are also reviewed.

\section{Case reports}

\section{CASE 1}

A 26 year old salesman with a lifelong history of mild eczema, presented with classical Philadelphia chromosome positive chronic myeloid leukaemia in 1975. Although he had massive splenomegaly and a presenting white cell count of $455 \times 10^{9} / 1$, his leukaemia was sensitive to busulphan and he remained well for a number of years. By 1983 increasing splenomegaly, resistance to busulphan, and additional 
chromosomal abnormalities-that is, $45 \mathrm{X},-\mathrm{Y}, 7 \mathrm{p}^{+}$, ring 17-indicated a more accelerated, malignant, phase of the disease.

In July 1984 he developed an ulcer, $2 \mathrm{~cm}$ in diameter, over the left medial malleolus. This was superficial with an undermined, raised, bluish-grey margin and an area of surrounding erythema. His white cell and platelet count at the time were $15.8 \times 10^{9} / 1$ and $212 \times 10^{9} / 1$, respectively, and he was taking no drugs. He appeared otherwise well, and there was no evidence of any other systemic disease. Swab culture from the ulcer base grew Staphylococcus aureus, but his response to flucloxacillin was transient and the ulcer failed to heal. In early September the ulcer became rapidly more extensive, increasing fourfold in size within three days (fig 1). Atypical bullous pyoderma gangrenosum was diagnosed. A biopsy specimen of the ulcer margin was consistent with pyoderma gangrenosum, showing a dense accumulation of polymorphs in the dermis but no evidence of vasculitis.

Treatment with prednisolone $(40 \mathrm{mg} /$ day $)$ was followed by a characteristically rapid response, ulcer healing being largely complete within two weeks. There was no recurrence of the ulcer as the dose of prednisolone was reduced progressively to $7.5 \mathrm{mg} / \mathrm{day}$, and he remained reasonably well until his $\underline{\underline{0}}$ sudden death from septicaemia four months later.

CASE 2

A 46 year old housewife presented with acute myeloid $\frac{\bar{C}}{\bar{N}}$ leukaemia, FAB classification M2, in February 1985. $\frac{\bar{\sigma}}{\bar{\sigma}}$ This was initially treated with two courses of standard $\stackrel{\otimes}{\AA}$ daunorubicin, cytosine arabinoside, and thioguanine ("3 + 10 DAT"), both of which were followed by an $\vec{\circ}$ unusually prolonged period of pancytopaenia. Remission was achieved in June 1985 and a consoli- $\vec{\omega}$ dation course of M-Amsa and VP16 was then given.

Two weeks later, when her neutrophil and platelet $\frac{\bar{g}}{8}$ count had fallen to $0.05 \times 10^{9} / 1$ and $10 \times 10^{9} / 1$, respectively, she developed Gram negative septicaemia with a fever of $39.5^{\circ} \mathrm{C}$ and widespread pur- $\omega_{\infty}$ pura. A slightly tender bluish-grey swelling, $1 \mathrm{~cm}$ in

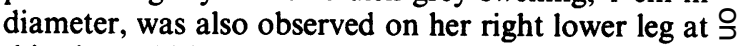
this time. Although this was thought to be caused either by infection or haemorrhage, its appearance $\frac{D}{0}$ remained unchanged, despite platelet transfusions and several days of intravenous piperacillin and gen- $\vec{\varphi}$ tamycin, even though both fever and purpura $\stackrel{\infty}{.}$ resolved rapidly. Two weeks later, when her neu-

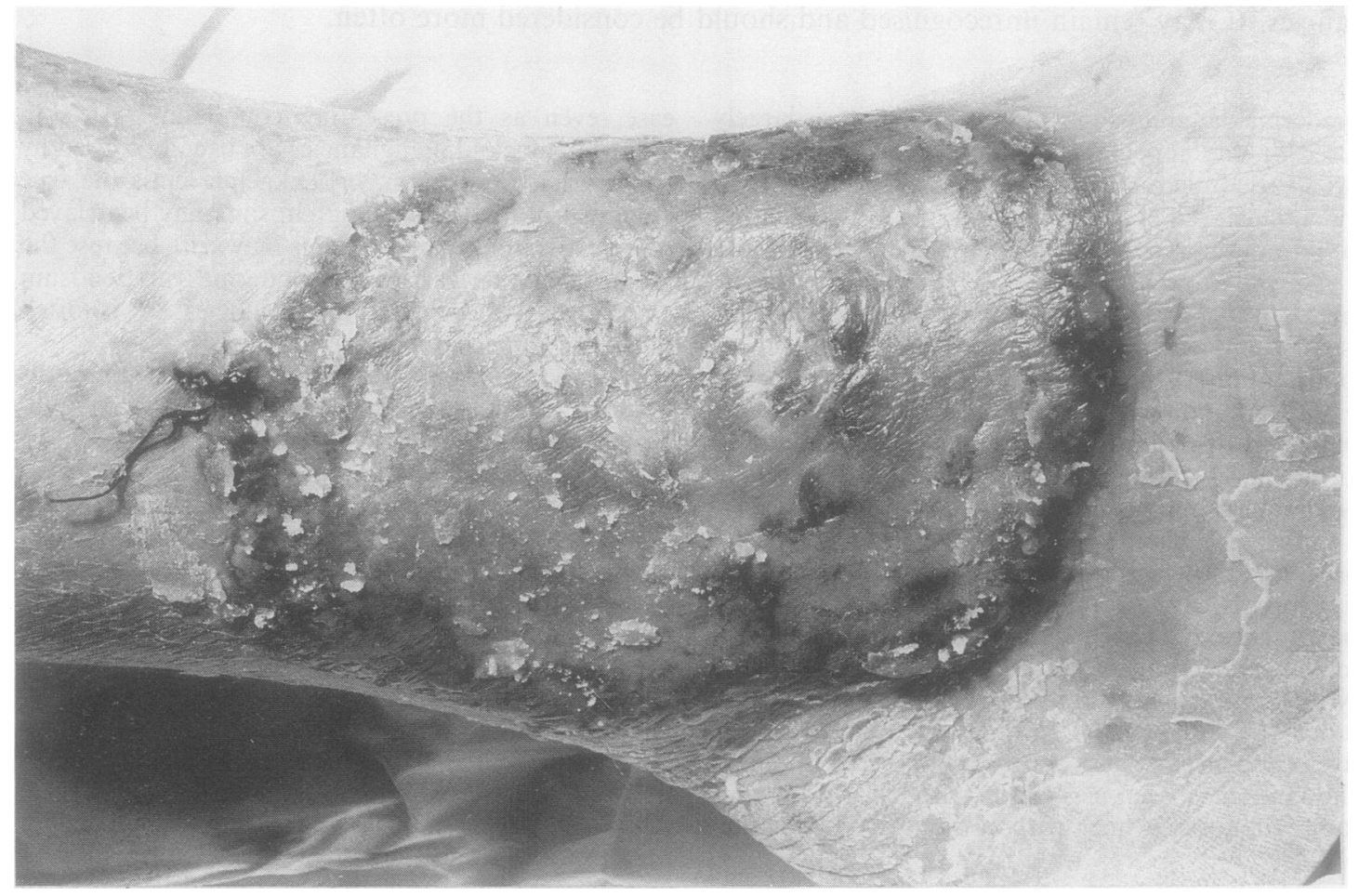

Fig 1 Rapidly extending ulcer over medial malleolus from case 1 showing bluish-grey undermined margin and surrounding erythema. 
trophil and platelet counts had risen to $0.8 \times 10^{9} / 1$ and $50 \times 10^{9} / 1$, this swelling extended rapidly and broke down. Its appearance was now that of a superficial circular ulcer, $4 \mathrm{~cm}$ in diameter, with an undermined, raised, bluish border and a surrounding halo of erythema. Swab cultures from the ulcer grew no pathogens. She was otherwise well with no evidence of any other systemic disease and taking only norethisterone and allopurinol at that time. Atypical bullous pyoderma gangrenosum was diagnosed, a skin biopsy specimen was obtained, and treatment with prednisolone $(40 \mathrm{mg} /$ day) started. She responded rapidly to prednisolone, almost complete healing occurring within two weeks. The microscopic appearance of the skin was unusual in some respects; the usual heavy dermal infiltration of neutrophils was absent, though a mononuclear infiltrate and some vasculitis and extravasation of erythrocytes was seen (figs 2 and 3). There was no evidence of leukaemic disease, fungal, or mycobacterial infection.

The dose of prednisolone was reduced by degrees and finally stopped six weeks later, without recurrence of her pyoderma gangrenosum. Unfortunately, after a further course of M-Amsa and VP16, she developed anti-HLA antibodies and, unresponsive to platelet transfusions, died from a haemorrhagic stroke.
CASE 3

Aged 65, this female patient was admitted in January 1985 with a two week history of a painful swollen right foot. Examination showed that she was clinically anaemic and feverish, with a temperature of $39 \cdot 5^{\circ} \mathrm{C}$. She was found to have several large and painful fluid-filled bullae, $3-8 \mathrm{~cm}$ in diameter, on the plantar and medial aspects of the right foot. These had a slightly raised pale bluish-grey margin and were surrounded by widespread erythema. Culture of the blister fluid grew only coagulase negative staphylococci, and blood culture was sterile at seven days. Further investigation showed a haemoglobin of $7.9 \mathrm{~g} / \mathrm{dl}$, a platelet count of $116 \times 10^{9} / 1$, and a neutrophilia of $11.5 \times 10^{9} / 1$. Bone marrow examination was consistent with refractory anaemia with excess of blasts (RAEB). Intravenous penicillin and gentamycin were started and her fever and confusion gradually resolved over the next few days. In contrast to her general improvement, the bullae extended and became confluent, breaking down to form a large superficial ulcer over much of the sole and medial aspect of the foot. Atypical bullous pyoderma gangrenosum was diagnosed and a skin biopsy specimen was obtained. The microscopic appearance of this was again non-specific but was consistent with the diagnosis. There was a heavy neutrophilic infiltration

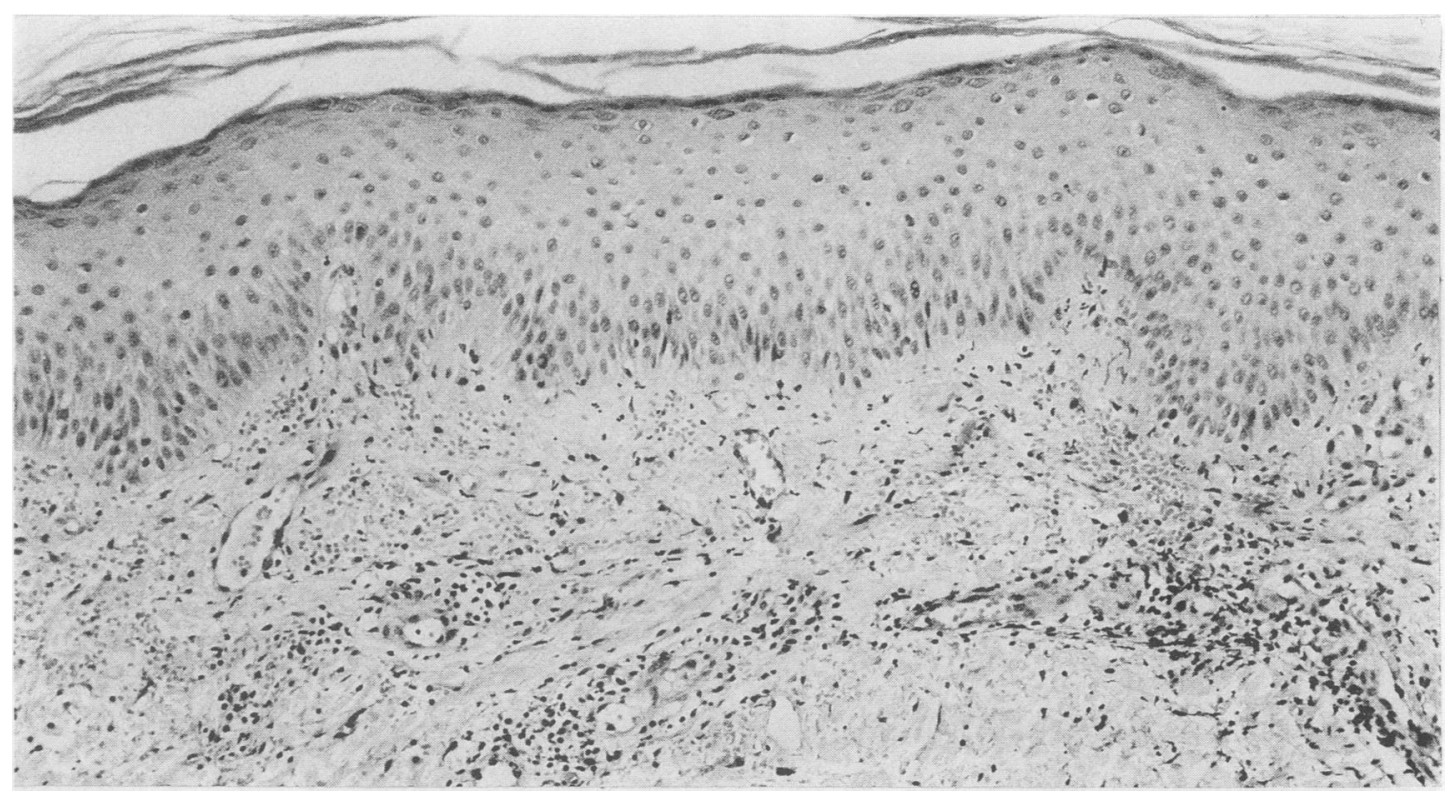

Fig 2 Low power photomicrograph of biopsy specimen from case 2. Lymphohistiocytic infiltrate is seen around vessels in papillary and reticular dermis. Mild degree of vascular damage is visible but severe extravasation of erythrocytes. No epidermal changes or polymorphs are seen (Haematoxylin and eosin.) 


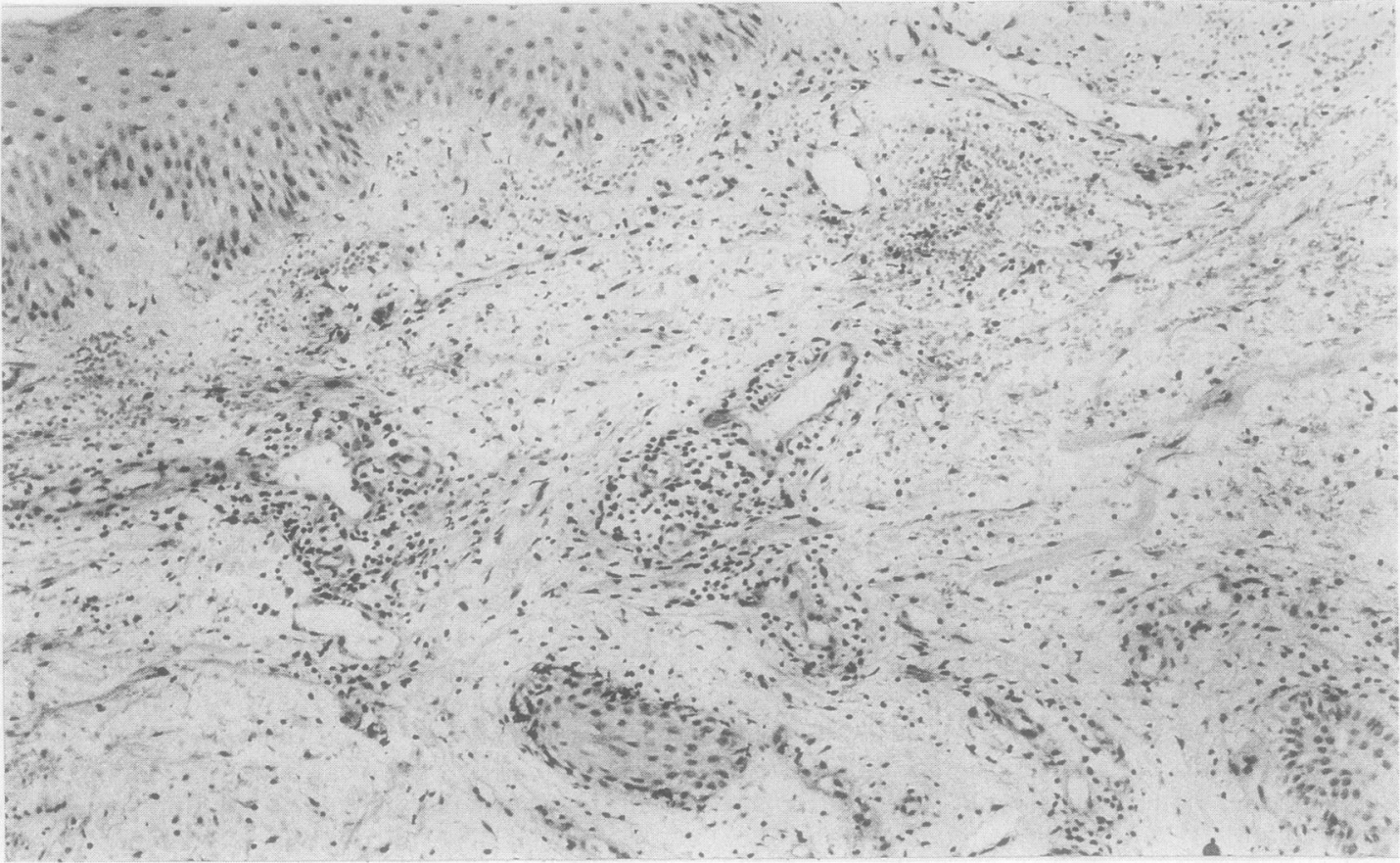

Fig 3 Higher power photomicrograph from case 2 showing perivascular infiltrate, extravasation of erythrocytes, and relative sparing of adnexal structures. Again, no polymorphs are seen (Haematoxylin and eosin.)

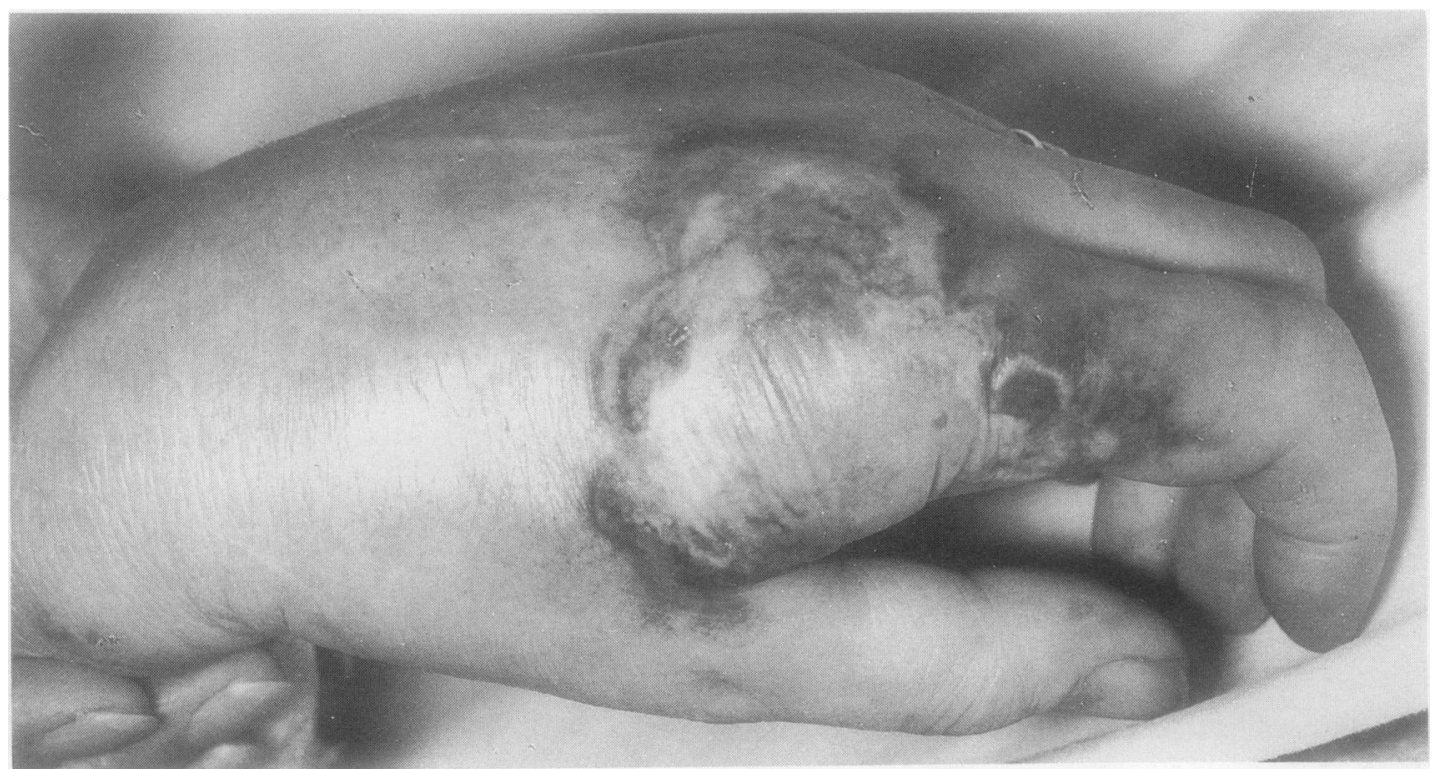

Fig 4 Painful bullus arising on back of left hand from case 3, photographed 36 hours after appearance of initial area of erythema, showing raised pale bluish-grey margin and surrounding erythema. 
of the dermis with no evidence of vasculitis, leukaemic infiltration, or infection with fungi or acid fast bacilli.

Treatment with prednisolone $(40 \mathrm{mg} /$ day) arrested further extension of the ulcer on her foot and was associated with gradual healing. Although her initial response to prednisolone was good, attempts to reduce the dose were associated with the appearance of new areas of pyoderma. These usually arose over pressure points and areas of mild trauma such as intravenous cannula sites. They began as areas of painful erythema extending rapidly and proceeding within 24 hours to bullae, with rapidly extending raised bluish-grey margins surrounded by advancing erythema. The bullae broke down to form painful superficial ulcers with undermined bluish-grey margins. Fig 4 shows a typical example arising on the back of the left hand. At whatever stage of evolution these lesions were, increasing the dose of prednisolone arrested further development and was followed slowly by healing.

Her course was marked by a series of episodes of septicaemia and an episode of pneumonia. There did not, however, seem to be any clear cut association between her infections and exacerbations of pyoderma gangrenosum, from which she was never entirely free. As old lesions healed, new ones appeared at irregular intervals. By September her RAEB had evolved into frank acute myeloid leukaemia. Her physical condition was poor and multiple chemotherapy was considered to be inappropriate. She deteriorated rapidly and died from bronchopneumonia in October 1985.

\section{Discussion}

The uncommon association between myeloid malignancies and pyoderma gangrenosum is now well documented, ${ }^{47}$ accounting for two of 86 patients in a recent series. ${ }^{4}$ The distribution of the skin lesions may be of some help in arriving at a diagnosis. The legs are the commonest site for the presenting lesion; $78 \%$ of patients in a recent series ${ }^{4}$ and all of ours were thus affected. Pyoderma gangrenosum often arises at the site of minor skin trauma, ${ }^{49}$ as in case 3 . The association between pyoderma gangrenosum and myeloid malignancies is often unrecognised, however, and several diagnostic difficulties, illustrated by our three cases, cause the diagnosis to be delayed or even missed. The difficulties arise firstly from the clinical context in which the lesions develop, and secondly from their atypical appearance. Most skin lesions in leukaemic patients are either haemorrhagic or infective, ${ }^{1}$ and our three cases were initially treated as such. The atypical bullous form of pyoderma gangrenosum is uncommon and may not be recognised, particularly when, as in case 3 , it is the presenting complaint and the skin lesion, therefore, seems to develop in the absence of an associated condition. In contrast with the classical form, atypical bullous pyoderma gangrenosum presents with concentric bullous areas spreading centrifugally and often rapidly. These break down to form ulcers which are relatively superficial unlike the deep destructive and penetrating ulcers sometimes seen in the classical form. The raised and undermined borders of the ulcers are a relatively subdued bluish-grey colour, in sharp contrast with the much brighter violaceous border usually associated with classical pyoderma gangrenosum. ${ }^{4-7}$

There has been considerable confusion concerning the histopathological changes. As a result, in cases of diagnostic difficulty, the histopathology is often of limited help and in our third case was actively misleading. Some authorities describe necrotising vasculitis $^{2}$ while others describe non-specific changes. ${ }^{3}$ Whereas some authors consider that vasculitis, as seen in case 2 , excludes the diagnosis, ${ }^{5}$ most authorities now accept that vasculitis is seen in a proportion of cases. ${ }^{34}$ Almost all reports emphasise the presence of a heavy mixed infiltrate of neutrophils and lymphocytes and small abcesses, characteristically seen in both the papillary and reticular dermis. ${ }^{124}$ This neutrophilic infiltrate varies in density according to the site of biopsy, being less dense if the biopsy is taken from the periphery of the lesion, as were all of ours, or from the base, where only chronic inflammatory cells may be seen. ${ }^{4}$ Similarly, in very early lesions the neutrophil component may be slight or even absent. Although neutrophils were absent in the biopsy specimen taken from our second case, this is not surprising as she was profoundly neutropenic at the time, and she was probably incapable of mounting a neutrophilic response. Clearly the patient's neutrophil count should be borne in mind when interpreting the appearance of such a biopsy. The absence of neutrophils from this biopsy also questions the causal association, suggested by some authors, between neutrophils, abnormal neutrophil function, and the development of pyoderma gangrenosum. ${ }^{9}$

Sweet's syndrome is also characterised by dermal infiltration with neutrophil polymorphs in the absence of recognisable infection and is associated with a similar range of conditions. ${ }^{110}$ Although it is clinically distinct in that it never ulcerates, these two skin lesions probably represent different ends of a clinical spectrum and may arise simultaneously in some patients. ${ }^{1}$ The purpose of the skin biopsy is clearly not to provide independent confirmation of pyoderma gangrenosum but to exclude other specific pathology as the microscopic appearance, although characteristic, is non-specific. Ulcers with a similar clinical appearance may be caused by systemic vasculitis, atypical my- 
cobacterial infection, deep fungal infections, facticial ulcers, and occasionally leukaemic skin infiltration, though the latter usually presents in a papular form. In the absence of an alternative diagnosis it is therefore unwise to exclude pyoderma gangrenosum on the basis of the microscopic appearance.

Once the diagnosis has been established, the response to prednisolone is usually rapid, as it was in our three cases. The response of atypical bullous gangrenosum may, indeed, be more rapid than that found with fully developed classical pyoderma as there is usually less tissue destruction.

Patients with myeloid malignancies who develop pyoderma gangrenosum have been observed to have a particularly poor prognosis. ${ }^{7}$ This probably relates in part to the underlying disease, particularly as the observation dates from a time when chemotherapy was less effective. Our patients also survived for only a short time. As pyoderma gangrenosum may be an immune complex disorder ${ }^{49}$ and as the presence of immune complexes has been related to poor prognosis in acute myeloid leukaemia ${ }^{11}$ it is tempting to hypothesise that immune complexes may be the aetiological link between atypical bullous pyoderma gangrenosum and poor prognosis myeloid malignancies. This would not, however, explain the occasional finding of pyoderma gangrenosum in patients with polycythaemia rubra vera. ${ }^{45}$

We have seen three cases in one year and, with the benefit of hindsight, can identify further cases which were not recognised at the time and which failed to respond to platelets and antibiotics. We therefore think that atypical bullous pyoderma is an uncommon but not a rare complication of myeloid malignancies and should be considered more often.

References

1 Caughman W, Stern R, Haynes H. Neutrophilic dermatosis of myeloproliferative disorders. J Am Acad Dermatol $\frac{\omega}{\vec{D}}$ 1983;9:751-8.

2 Samitz MH, Dana AS, Rosenberg P. Cutaneous vasculitis in association with Crohn's disease. Cutis 1970;6:51-6.

3 Lever WF, Shaumberg-Lever G. Histopathology of the skin. 6th $\overrightarrow{0}$ ed. Philadelphia: JB Lippincott Company, 1983:194-6.

4 Powell FC, Schroeter AL, Su WPD, Perry HO. Pyoderma gangrenosum: a review of 86 patients. $Q J$ Med 1985;55:173-86. W

5 Romano J, Safai B. Pyoderma gangrenosum and myeloproliferative disorders: report of a case and review of the litera- $\frac{}{0}$ ture. Arch Intern Med 1979;139:932-4.

6 Collen JP, Dubin AV, Gehrke CF. Recurrent pyoderma gangren- . osum and agnogenic myeloid metaplasia. Arch Dermatol 1977;113:1585-6.

7 Perry HO, Winkelmann RK. Bullous pyoderma gangrenosum and leukaemia. Arch Dermatol 1972;106:901-5.

8 Beurey J, Canton P, Weber M, et al. Pyoderma gangrenosum cervical: traitment par echanges plasmatique. Ann Dermatol $D$ Veneriol 1982;109:455-9.

9 Brunsting LA, Goeckerman WH, O'Leary PA. Pyoderma (ecthyma) gangrenosum: clinical and experimental observations in $\overrightarrow{0}$ five cases occurring in adults. Arch Dermatol 1930;22:655-80.

10 Cooper PH, Innes DJ, Greer KE. Acute febrile neutrophilic der-. matosis (Sweet's syndrome) and myeloproliferative disorders. Cancer 1983;51:1518-26.

11 Carpentier NA, Fiere DM, Schuh D, Ghislaine T, Lange TA, Lambert $\mathrm{PH}$. Circulating immune complexes and the prognosis of acute myeloid leukaemia. $N$ Engl J Med 1982;307:1174-80.

Requests for reprints to: Dr CRM Hay, University Department of Haematology, Royal Hallamshire Hospital, Glossop Road, Sheffield S10 2JF, England. 